\title{
Cruising through molecular bound-state manifolds with radiofrequency
}

\author{
F. LANG' ${ }^{1}$, P. V. D. STRATEN ${ }^{2}$, B. BRANDSTÄTTER ${ }^{1}$, G. THALHAMMER ${ }^{1}$, K. WINKLER ${ }^{1}$, P. S. JULIENNE ${ }^{3}$, \\ R. GRIMM ${ }^{1,4}$ AND J. HECKER DENSCHLAG'* \\ ${ }^{1}$ Institut für Experimentalphysik und Forschungszentrum für Quantenphysik, Universität Innsbruck, 6020 Innsbruck, Austria \\ ${ }^{2}$ Debye Institute, Universiteit Utrecht, 3508 TA Utrecht, Netherlands \\ ${ }^{3}$ Atomic Physics Division and Joint Quantum Institute, National Institute of Standards and Technology, Gaithersburg, Maryland 20899, USA \\ ${ }^{4}$ Institut für Quantenoptik und Quanteninformation, Österreichische Akademie der Wissenschaften, 6020 Innsbruck, Austria \\ *e-mail: Johannes.Denschlag@uibk.ac.at
}

Published online: 27 January 2008; doi:10.1038/nphys838

The production of ultracold molecules with their rich internal structure is currently attracting considerable interest ${ }^{1-4}$. For future experiments, it will be important to efficiently transfer these molecules from their initial internal quantum state at production to other quantum states of interest. Transfer tools such as optical Raman schemes ${ }^{5,6}$, radiofrequency transitions (see, for example, ref. 7) or magnetic field ramping ${ }^{8,9}$ exist, but are either technically involved or limited in their applicability. Here, we demonstrate a simple, highly efficient hybrid transfer method that overcomes a number of the previous limitations. The scheme is based on magnetically tuned mixing of two neighbouring molecular levels, which enables otherwise forbidden radiofrequency transitions between them. By repeating this process at various magnetic fields, molecules can be successively transported through a large manifold of quantum states. Applying nine transfers, we convert very weakly bound Feshbach molecules to a much more deeply bound level with a binding energy corresponding to $3.6 \mathrm{GHz}$. As an important spin-off of our experiments, we demonstrate a high-precision spectroscopy method for investigating level crossings.

Radiofrequency has important applications for ultracold molecules, such as spectroscopy ${ }^{7,10-14}$ and molecule production ${ }^{15-17}$. Using radiofrequency to transfer ground-state molecules between states of different vibrational quantum numbers, as demonstrated here, is not obvious. For simple molecular potentials, transition matrix elements for magnetic dipole transitions between different vibrational levels are expected to vanish on the basis of an overlap argument of the spatial wavefunctions. However, for real molecules such as $\mathrm{Rb}_{2}$, the situation is more complex, for example, owing to exchange interaction, hyperfine structure and the Zeeman effect. The combined effect of these interactions induces mixing of states with different vibrational quantum numbers, leading to new eigenstates between which radiofrequency transitions can be driven (see the Methods section). This mixing effect is maximal at avoided crossings. (As a consequence, it is important for radiofrequency spectroscopy at Feshbach resonances ${ }^{12}$.)

We carry out our experiments with a pure, ultracold ensemble of $2 \times 10^{4}$ ultracold ${ }^{87} \mathrm{Rb}_{2}$ Feshbach molecules, each of which is held in an individual optical microtrap (see the Methods section). Figure 1 shows the relevant molecular level spectrum for our experiment as calculated by a coupled-channel model ${ }^{18,19}$ based on adjusted $a b$ initio $\mathrm{Rb}_{2}$ Born-Oppenheimer potentials ${ }^{20}$. It essentially consists of straight lines of $s$ - and $d$-wave levels (corresponding to a rotational angular momentum $l=0,2$, respectively). In general, at magnetic fields where two levels intersect, coupling between them gives rise to an avoided crossing.

In the following, we will use the level spectrum like a street map, as the molecules move through the manifold of molecular bound states by sweeping the magnetic field. When arriving at a level intersection we can turn off or go straight, traversing the avoided crossing. In principle, the avoided crossing can be jumped via a fast magnetic field ramp ${ }^{8,9}$. This, however, is limited to very small splittings (typically $<200 \mathrm{kHz} \times h$ ) owing to practical limitations of the controllable magnetic ramp speed. This constraint can be easily overcome using a radiofrequency transition as we demonstrate below.

As an example for cruising through molecular bound-state levels, we choose the diagonal path in Fig. 1, as marked with the red circles $A$ to $K$, each indicating an avoided crossing. This converts our Feshbach molecules with their weak binding energy of $24 \mathrm{MHz} \times h$ to a deeper bound level at zero magnetic field, $3.6 \mathrm{GHz} \times h$ below the $f=1, m_{\mathrm{f}}=1$ dissociation limit.

Figure 2a shows an expanded view of the first avoided crossing A. The upper branch is connected to the Feshbach resonance at $1,007.4 \mathrm{G}$ and is initially populated with Feshbach molecules at point i. We use adiabatic passage as a very efficient way for population transfer to the lower branch. We switch on a magnetic radiofrequency field which couples the upper and lower branches, inducing two narrow avoided crossings between the dressed states (Fig. 2b). An adiabatic magnetic field sweep to point ii and subsequent switching off the radiofrequency completes the transfer (see the Methods section).

Figure $2 \mathrm{~d}$ shows the fraction $N / N_{0}$ of remaining molecules in the upper branch after the transfer to the lower branch (diamonds) as a function of the radiofrequency field amplitude $B_{\mathrm{rf}}$. For sufficiently high amplitude, no more molecules are detected (see the Methods section). To verify that molecules are not simply lost for high amplitude, we also carry out a transfer back (ii $\rightarrow$ i) to the upper branch (circles). All molecules can be recouped again for strong enough radiofrequency fields.

From similar measurements with repeated transfers between the upper and lower branches, we infer single transfer efficiencies 


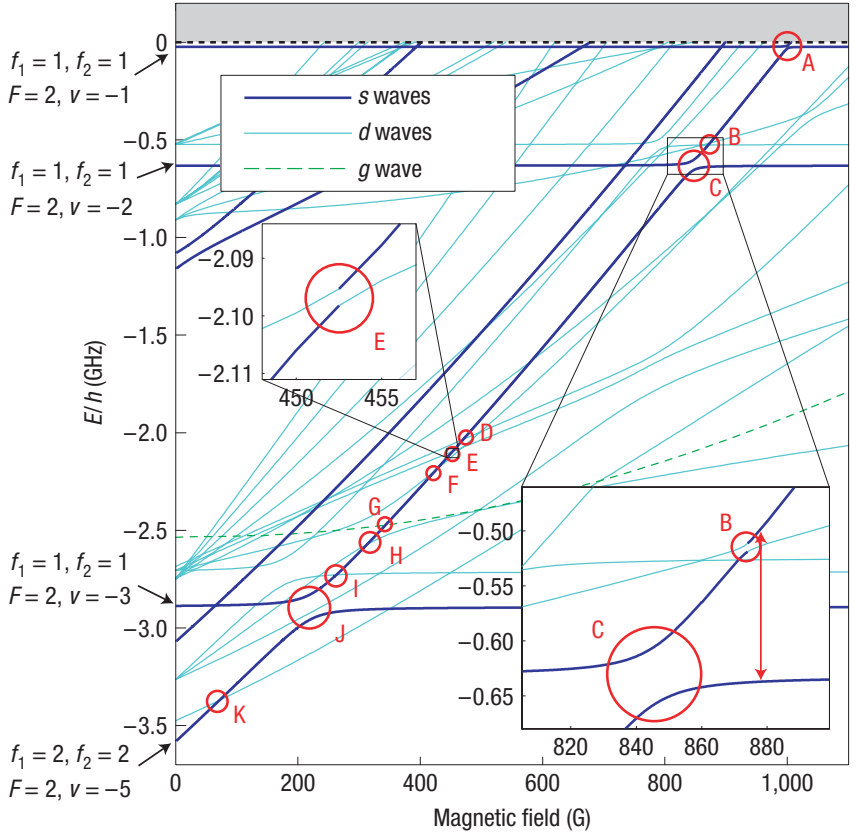

Figure 1 Path through manifold of molecular levels. Energy spectrum of relevant molecular levels of ${ }^{87} \mathrm{Rb}_{2}$ in the electronic ground state with $m_{\mathrm{Ftot}}=2$. The zero of energy is taken to be that of two separated atoms at each field strength and marks the dissociation threshold for a pair of $f=1 ; m_{\mathrm{f}}=1$ ground-state atoms. Molecules are transported through the bound-level manifold by traversing avoided crossings, marked $\mathrm{A}-\mathrm{K}$. These levels are characterized by their respective quantum numbers at zero magnetic field, that is, global angular momentum $F_{\text {tot }}, m_{\mathrm{Ftot}}=2$, the angular momenta $f_{1}, f_{2}$ of the atomic constituents, their combined angular momentum $F$ and the vibrational quantum number $v$. Out of the number of existing $g$-wave levels, we only show the single relevant one $(1 \mathrm{G}=0.1 \mathrm{mT})$.

of up to $99.5 \%$. Our experimental data are well fitted with the well-known Landau-Zener model ${ }^{21}$ (solid lines) where the transfer probability for a single transition is given by $1-\exp \left(-\pi \omega_{\mathrm{R}}^{2} \hbar / 2|\dot{B}|\left|\mu_{2}-\mu_{1}\right|\right)$. Here $\omega_{\mathrm{R}}$ is the Rabi frequency, $|\dot{B}|$ is the ramp speed and $\mu_{1}, \mu_{2}$ are the magnetic moments of the two states.

After this successful demonstration at crossing A, we will use such adiabatic transfers across avoided crossings (ATAC) repeatedly for traversing the remaining crossings on our path. We typically find avoided crossings to lie within a few Gauss of their predicted magnetic field position based on the coupled-channel calculation, which is within the accuracy of our model. This identification also helps us to verify that the molecules are in the right quantum level during transport (see the Methods section).

After traversing A, the next wide $s$-wave crossing is $\mathrm{C}$. Before we get to $\mathrm{C}$, however, we hit the avoided crossing B at $874 \mathrm{G}$ with a $\sim 7 \mathrm{MHz} \times h$ splitting, on the basis of an intersecting $d$-wave level (see Fig. 1 inset). To circumvent crossing B, we carry out the ATAC transfer between the $s$-waves levels already at $876 \mathrm{G}$, far from the $s$-wave crossing. This raises the question, how far from an avoided crossing the radiofrequency transitions can still be driven. Figure $3 \mathrm{a}$ is a zoom into the energy spectrum showing the avoided crossings $\mathrm{A}$ and $\mathrm{C}$. Figure $3 \mathrm{~b}$ shows the corresponding calculated magnetic dipole matrix elements $\mu_{\mathrm{u}, 1}$ between the corresponding upper and lower level branches using the coupled-channel model (see also the Methods section). The matrix elements are clearly peaked at their respective crossing, reaching values of more than a Bohr magneton. Such high coupling strengths are in agreement a

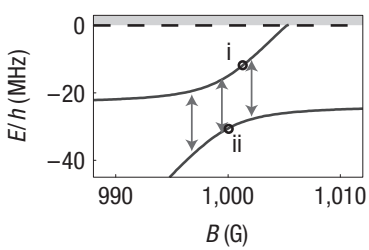

c

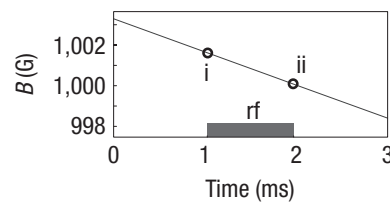

b.

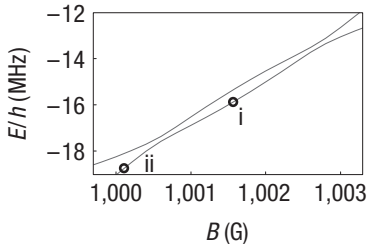

d

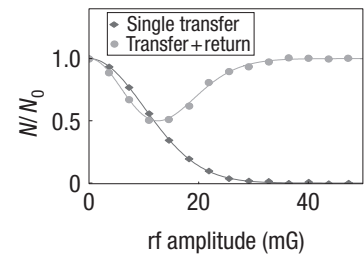

Figure 2 Adiabatic population transfer across avoided crossing with radiofrequency. a, Zoom into avoided crossing A of Fig. 1. Transitions between the upper and lower branches can be induced with radiofrequency. $\mathbf{b}$, Dressed-state picture. Coupling the lower and upper branches in a with blue-detuned radiofrequency induces two avoided crossings. c, Experimental transfer sequence (rf: radiofrequency). d, Measured transfer efficiency for transfer from the upper branch to the lower branch (diamonds) and back (circles). The solid lines are fits to a Landau-Zener model. The radiofrequency amplitude is calibrated with a global uncertainty of about $40 \%$.

a

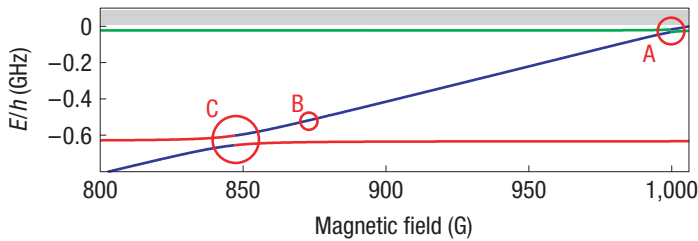

b

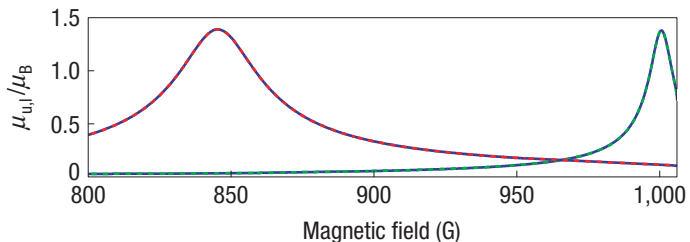

Figure 3 Calculated magnetic dipole matrix elements for the avoided crossings A and $\mathbf{C}$ of Fig. 1. a,b, Colour coding identifies the levels (a) with their respective transition (b).

with our measurements in Fig. $2 \mathrm{~d}$, where $\mu_{\mathrm{u}, 1}$ can be extracted from the fits by using $\omega_{\mathrm{R}}=B_{\mathrm{rf}} \mu_{\mathrm{u}, 1}$ and measuring $B_{\mathrm{rf}}$. The width of the peaks scales with the energy splitting of their avoided crossing. When moving away from the crossing at $B_{0}$, the matrix elements vanish inversely proportional to $\left|B-B_{0}\right|$ (see the Methods section).

Continuing our path down by lowering the magnetic field, we hit consecutively five avoided crossings (D,E,F,H,I) with $d$-wave states. The corresponding energy splittings are of the order of $1 \mathrm{MHz} \times h$ and are each crossed by the ATAC method, which demonstrates its universal character. In general, however, ATAC transfers at narrow avoided crossings are technically more challenging owing to a small magnetic field range of strong coupling and thus a greater susceptibility to magnetic field noise which can lead to unwanted non-adiabatic transitions. Coupling to a $g$-wave state is observed as well (crossing G), but it is weak enough to be overcome by diabatic ramping of the magnetic field. Finally, after crossing $\mathrm{J}$ and $\mathrm{K}$ we reach zero magnetic field, with 


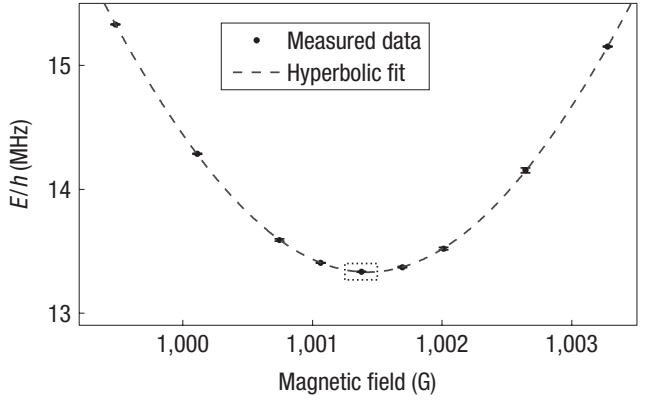

Figure 4 Spectroscopy of an avoided crossing. For the avoided crossing A, the splitting is measured for various magnetic fields by determining the resonant transition frequency using a lorentzian fit. The error bars represent a $95 \%$ confidence interval for the centre position of the lorentzian and are typically smaller than the size of the plot symbol. The dashed line is a hyperbolic fit yielding a minimum frequency of $(13.331 \pm 0.005) \mathrm{MHz}$.

the molecules in state $\left|l=0, F_{\text {tot }}=F=f_{1}=f_{2}=m_{\text {Ftot }}=2, v=-5\right\rangle$, $3.6 \mathrm{GHz}$ below the $f_{1}=f_{2}=1, m_{\mathrm{f} 1}=m_{\mathrm{f} 2}=1$ threshold. We have also produced $d$-wave molecules at zero magnetic field $\left(\left|l=2, F_{\text {tot }}=2, F=0, f_{1}=f_{2}=m_{\text {Ftot }}=2, v=-5\right\rangle\right)$ by adiabatically following the upper branch in crossing $\mathrm{K}$, that is, taking a right turn. The complete transfer down across all 10 avoided crossings takes about $90 \mathrm{~ms}$ with a global transfer efficiency of about $50 \%$. The losses during transfer can be explained mainly by the limited molecular lifetime of $280 \mathrm{~ms}$ in the lattice, due to inelastic scattering of lattice photons ${ }^{22}$, and by not fully optimized transfers at several crossings.

We also developed a high-precision spectroscopy method for measuring the minimal energy splitting of an avoided crossing. For a given avoided crossing, the energy splitting is measured for various magnetic fields. We use two methods. Method 1 determines the resonance frequency for transfer of molecules between the two branches of the avoided crossing. Using a single radiofrequency pulse of a few milliseconds length, we look for the frequency of maximal transfer. The corresponding data for crossing A are shown in Fig. 4 and are very well fitted by a hyperbolic curve, yielding a splitting of $(13.331 \pm 0.005) \mathrm{MHz} \times h$.

To increase the precision we use method 2 , where we carry out a Ramsey-type interferometric measurement (Fig. 5a). A $\pi / 2$-pulse of radiofrequency transfers $50 \%$ of the Feshbach molecules to the lower branch, creating a 50/50 coherent superposition. After a hold time $t_{\mathrm{h}}$ and a second $\pi / 2$-pulse, the number of Feshbach molecules is detected. We observe an oscillation of this population $N$ (Fig. 5b) that corresponds precisely to the detuning of the radiofrequency field from resonance. Coherence times reach $1 \mathrm{~ms}$. Figure $5 c$ shows a set of data taken in the region indicated by the dotted box in the centre of Fig. 4. The clear deviation from the hyperbolic curve results from a $\sim 2 \mathrm{G} \mathrm{mm}^{-1}$ magnetic field gradient across the molecular cloud ( $\sim 20 \mu \mathrm{m}$ diameter $)$ in combination with $\sim 20 \mathrm{mG}$ fluctuations of the magnetic field during the time of a scan. This behaviour is reproduced by our model calculation (solid line) taking these experimental imperfections into account. From the model, we obtain a best estimate of the minimum splitting of $(13.33210 \pm 0.00015) \mathrm{MHz} \times h$ for the ideal hyperbolic curve. The upshift of about $150 \mathrm{~Hz}$ of the minimum of the model curve with respect to the hyperbolic curve is due to averaging over the magnetic field inhomogeneities.

We have also carried out detailed measurements of the energy splitting at the avoided crossings marked C, E and J in Fig. 1. These data are shown in Supplementary Information, Table S1.
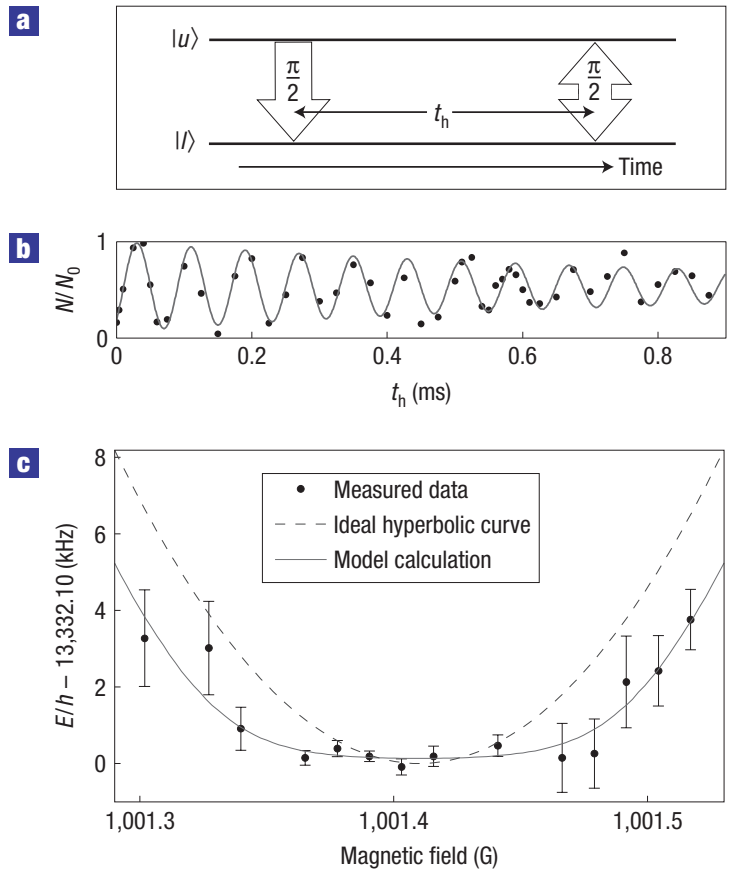

Figure 5 Spectroscopy with Ramsey interferometry. a, Ramsey scheme consisting of two $\pi / 2$-pulses of radiofrequency and a holding time $t_{\mathrm{h}}$. $\mathbf{b}$, Fraction of remaining Feshbach molecules after a Ramsey measurement as holding time is varied (here, $B=1,001.39 \mathrm{G}$ ). The oscillation frequency corresponds to the detuning of the radiofrequency from the splitting and is determined by fitting an exponentially damped oscillation to the data. c, Measured splittings, in a narrow magnetic field region (indicated by the small dotted box in Fig. 4). The deviation from the hyperbolic curve (dashed line) results from magnetic field fluctuations and inhomogeneities and is reproduced by our model calculation (solid line). The error bars indicate a $95 \%$ confidence interval for the fit (b).

The measurements are complementary to conventional boundstate spectroscopy because instead of measuring the plain energy spectrum of the bound states, our method determines the strength of the coupling between levels. The precision of our data is several orders of magnitude better than the accuracy of our current coupled-channel model. Thus, the data can serve to improve and test the theoretical models used to calculate molecular energy levels.

To conclude, we have demonstrated an efficient method (ATAC) to transfer molecules between neighbouring quantum states. It works for any kind of molecule, whether tightly or loosely bound, as long as the two levels for transfer exhibit a magnetically tunable avoided crossing in an accessible magnetic field range. For weakly bound $\mathrm{Rb}_{2}$ molecules, our experiments highlight ATAC transfers between different vibrational levels. For tightly bound triplet $\mathrm{Rb}_{2}$ levels, ATAC will be particularly useful for transfer between states of different rotational angular momentum $l$ (see also Supplementary Information). This opens interesting perspectives for experiments in cold collisions ${ }^{23-25}$, chemistry in the ultracold regime, high-resolution spectroscopy, matter-wave interferometry and molecular Bose-Einstein condensates. In particular, we plan to use the ATAC method to prepare Feshbach molecules in a convenient start position for an optical Raman transition to a deeply bound molecular state or even the vibrational ground state $^{6,26}$, optimizing in this way Franck-Condon factors and stability issues. Furthermore, the ATAC scheme can be extended in a straightforward manner to avoided crossings that are tuned by electrical fields. 


\section{METHODS}

\section{PREPARATION OF FESHBACH MOLECULES IN MICROTRAPS}

We load a Bose-Einstein condensate of ${ }^{87} \mathrm{Rb}$ atoms into the lowest Bloch band of a deep cubic three-dimensional optical lattice with lattice period $415 \mathrm{~nm}$. After the atoms are prepared in state $f=1, m_{\mathrm{f}}=1$, ramping over a Feshbach resonance at 1,007.4 $\mathrm{G}$ (ref. 27) $(1 \mathrm{G}=0.1 \mathrm{mT})$ produces Feshbach molecules. A final purification step removes all chemically unbound atoms $\mathrm{s}^{22}$. There is no more than a single molecule per lattice site and the lattice potential is deep enough $\left(\approx 10 \mu \mathrm{K} \times k_{\mathrm{B}}\right)$ to effectively isolate the molecules from each other, shielding them from detrimental collisions ${ }^{22}$. Thus, the sites can be viewed as microtraps holding single molecules in the trap ground state.

\section{ADIABATIC TRANSFER ACROSS AN AVOIDED CROSSING}

Exemplarily for all ATAC transfers, we discuss here in detail the transfer at avoided crossing A (Fig. 2a). At the beginning of the transfer sequence, the radiofrequency field is off (Fig. 2c). The Feshbach molecules are brought to point $\mathrm{i}$ by ramping the magnetic field to $B=1,001.4 \mathrm{G}$. We then switch on a longitudinal radiofrequency magnetic field (that is, it points in the same direction as the magnetic bias field) which couples the upper and lower branches of the avoided crossing via a magnetic dipole transition. We use a frequency of $13.6 \mathrm{MHz}$, which is blue-detuned with respect to the minimal splitting of the avoided crossing of about 13.3 MHz. In the dressed-state picture, the radiofrequency field with field amplitude of $B_{\mathrm{rf}} \sim 50 \mathrm{mG}$ produces two radiofrequency-induced avoided crossings, one above and one below point $\mathrm{i}$ (Fig. 2b). Each avoided crossing shows an energy splitting corresponding to the Rabi frequency $\omega_{\mathrm{R}} \sim 2 \pi \times 70 \mathrm{kHz}$. Further lowering the magnetic field adiabatically, we reach point ii at $1,001.1 \mathrm{G}$ after $1 \mathrm{~ms}$. Subsequent switching off the radiofrequency field completes the transfer of the molecules to the lower branch.

\section{DETECTION OF MOLECULES AND THEIR QUANTUM STATE}

To detect the molecules at any stage during their transport through the manifold of molecular levels, we trace back exactly the path we have come before, adiabatically traversing all avoided crossings in the opposite direction. We end up with Feshbach molecules that are dissociated into unbound atoms by sweeping over the Feshbach resonance at 1,007.4 G. These atoms are then counted via standard absorption imaging, after switching off the optical lattice and the bias magnetic field.

We use two methods to verify that molecules are in the right quantum level during transport. (1) Checking for consistency between predicted and experimentally found avoided crossings, in terms of magnetic field location and energy splitting. (2) Optical spectroscopy to measure the binding energy of molecules. By irradiating the molecules with resonant laser light, we transfer them to an electronically excited molecular level, $\left|0_{g}^{-}, v=31, J=0\right\rangle$ (ref. 6), leading to losses. The shift of this laser frequency compared with the frequency of the photoassociation transition to the same excited molecular level corresponds to the binding energy of the molecules.

\section{SIMPLE MODEL FOR RADIOFREQUENCY TRANSITIONS AT AN AVOIDED CROSSING}

A simple two-level model gives insight into the mechanism of the radiofrequency transitions at the avoided crossing. Two molecular bare levels $|b 1\rangle,|b 2\rangle$ with magnetic moments $\mu_{1}$ and $\mu_{2}$ cross at a magnetic field $B=B_{0}$. The hamiltonian for these levels reads

$$
\hat{H}=\left(B-B_{0}+B_{\mathrm{rf}} \cos \left(\omega_{\mathrm{rf}} t\right)\right)\left(\begin{array}{cc}
\mu_{1} & 0 \\
0 & \mu_{2}
\end{array}\right)+\frac{\hbar}{2}\left(\begin{array}{cc}
0 & \Omega \\
\Omega & 0
\end{array}\right) .
$$

A coupling $\Omega$ between the two levels, for example, due to exchange interaction or dipole-dipole interaction, leads to mixing and the new eigenstates $|u\rangle$ and $|l\rangle$. These states form the upper and lower branches of an avoided crossing, similar to Fig. 2a. A longitudinal magnetic radiofrequency field with amplitude $B_{\mathrm{rf}}$ and frequency $\omega_{\mathrm{rf}}$ can drive transitions between levels $|u\rangle$ and $|l\rangle$ which read

$$
\begin{gathered}
|u\rangle=\cos (\theta)|b 1\rangle+\sin (\theta)|b 2\rangle \\
|l\rangle=-\sin (\theta)|b 1\rangle+\cos (\theta)|b 2\rangle,
\end{gathered}
$$

with mixing angle $\theta=\arctan \left(\left(\delta+\sqrt{\delta^{2}+\Omega^{2}}\right) / \Omega\right)$, where $\delta=\left(\left(\mu_{2}-\mu_{1}\right)\left(B-B_{0}\right)\right) / \hbar$. The matrix element for the radiofrequency transition is then

$$
\begin{aligned}
\mu_{\mathrm{u}, 1} & \equiv\left\langle u\left|\left(\begin{array}{cc}
\mu_{1} & 0 \\
0 & \mu_{2}
\end{array}\right)\right| l\right\rangle=\left(\mu_{2}-\mu_{1}\right) \sin (2 \theta) \\
& =2\left(\mu_{2}-\mu_{1}\right) \frac{\Omega\left(\delta+\sqrt{\delta^{2}+\Omega^{2}}\right)}{\Omega^{2}+\left(\delta+\sqrt{\delta^{2}+\Omega^{2}}\right)^{2}} .
\end{aligned}
$$

Thus, $\mu_{\mathrm{u}, 1}$ is resonantly peaked at the avoided crossing with a width (full-width at half-maximum) of $2 \sqrt{3} \Omega$ and vanishes as $1 /\left(B-B_{0}\right)$ far away from the crossing.

We find good agreement when comparing the matrix elements of our simple model to the ones of the coupled-channel model, given by $\mu_{\mathrm{u}, 1}=\left\langle u\left|\mu_{\mathrm{B}} g_{\mathrm{s}} S_{\mathrm{z}}+\mu_{\mathrm{N}} g_{\mathrm{I}} I_{\mathrm{z}}\right| l\right\rangle$. Here $|u\rangle,|l\rangle$ are the wavefunctions as calculated with the coupled-channel model. $\mu_{\mathrm{B}}, \mu_{\mathrm{N}}$ are the Bohr magneton and nuclear magneton, $g_{\mathrm{s}}$ and $g_{\mathrm{I}}$ are the $g$-factors of the electrons and nuclei, respectively, and $S_{z}, I_{z}$ are the corresponding spin operator components in the direction of the magnetic field.

\section{Received 30 August 2007; accepted 18 December 2007; published 27 January 2008.}

\section{References}

1. Doyle, J., Friedrich, B., Krems, R. V. \& Masnou-Seeuws, F. Quo vadis, cold molecules? Eur. Phys. J. D 31, 149-164 (2004).

2. Jones, K. M., Tiesinga, E., Lett, P. D. \& Julienne, P. S. Ultracold photoassociation spectroscopy: Long-range molecules and atomic scattering. Rev. Mod. Phys. 78, 483-535 (2006).

3. Köhler, T., Goral, K. \& Julienne, P. S. Production of cold molecules via magnetically tunable Feshbach resonances. Rev. Mod. Phys. 78, 1311-1361 (2006).

4. Special issue on cold molecules. J. Phys. B 39, S813-S1280 (2006).

5. Bergmann, K., Theuer, H. \& Shore, B. W. Coherent population transfer among quantum states of atoms and molecules. Rev. Mod. Phys. 70, 1003-1025 (1998).

6. Winkler, K. et al. Coherent optical transfer of Feshbach molecules to a lower vibrational state. Phys Rev. Lett. 98, 043201 (2007)

7. Bartenstein, M. et al. Precise determination of ${ }^{6} \mathrm{Li}$ cold collision parameters by radio-frequency spectroscopy on weakly bound molecules. Phys. Rev. Lett. 94, 103201 (2005).

8. Mark, M. et al. Stückelberg interferometry with ultracold molecules. Phys. Rev. Lett. 99, 113201 (2007).

9. Mark, M. et al. Spectroscopy of ultracold, trapped cesium Feshbach molecules. Phys. Rev. A 76, 042514 (2007).

10. Regal, C. A., Ticknor, C., Bohn, J. L. \& Jin, D. S. Creation of ultracold molecules from a Fermi gas of atoms. Nature 424, 47-50 (2003).

11. Chin, C. et al. Observation of the pairing gap in a strongly interacting Fermi gas. Science 305, $1128-1130$ (2004)

12. Chin, C. \& Julienne, P. Radio-frequency transitions on weakly bound ultracold molecules. Phys. Rev. A 71, 012713 (2005)

13. Köhl, M., Moritz, H., Stöferle, T., Günter, K. \& Esslinger, T. Fermionic atoms in a three-dimensional optical lattice: Observing Fermi surfaces, dynamics and interactions. Phys. Rev. Lett. 94, 080403 (2005).

14. Schunck, C. H., Shin, Y., Schirotzek, A., Zwierlein, M. W. \& Ketterle, W. Pairing without superfluidity: The ground state of an imbalanced Fermi mixture. Science 316, 867-870 (2007).

15. Thompson, S. T., Hodby, E. \& Wieman, C. E. Ultracold molecule production via a resonant oscillating magnetic field. Phys. Rev. Lett. 95, 190404 (2005).

16. Ospelkaus, C. et al. Ultracold heteronuclear molecules in a 3D optical lattice. Phys. Rev. Lett. 97, 120402 (2006).

17. Bertelsen, J. F. \& Mølmer, K. Molecule formation in an optical lattice by resonantly modulated magnetic fields. Phys. Rev. A 73, 013811 (2006).

18. Dulieu, O. \& Julienne, P. S. Coupled channel bound states calculations for alkali dimers using the Fourier grid method. J. Chem. Phys. 103, 60-66 (1995).

19. Tiesinga, E., Williams, C. J. \& Julienne, P. S. Photoassociative spectroscopy of highly excited vibrational levels of alkali dimers: Greens function approach for eigenvalue solvers. Phys. Rev. A 57, 4257-4267 (1998).

20. Krauss, M. \& Stevens, W. J. Effective core potentials and accurate energy curves for $\mathrm{Cs}_{2}$ and other alkali diatomics. J. Chem. Phys. 93, 4236-4242 (1990).

21. Vitanov, N. V. \& Garraway, B. M. Landau Zener model: Effects of finite coupling duration. Phys. Rev. A 53, 4288-4304 (1996)

22. Thalhammer, G. et al. Long-lived Feshbach molecules in a 3D optical lattice. Phys. Rev. Lett. 96, 050402 (2006).

23. Chin, C. et al. Observation of Feshbach-like resonances in collisions between ultracold molecules Phys. Rev. Lett. 94, 123201 (2005).

24. Staanum, P., Kraft, S. D., Lange, J., Wester, R. \& Weidemüller, M. Experimental investigation of ultracold atom-molecule collisions. Phys. Rev. Lett. 96, 023201 (2006).

25. Zahzam, N., Vogt, T., Mudrich, M., Comparat, D. \& Pillet, P. Atom-molecule collisions in an optically trapped gas. Phys. Rev. Lett. 96, 023202 (2006).

26. Jaksch, D., Venturi, V., Cirac, J. I., Williams, C. J. \& Zoller, P. Creation of a molecular condensate by dynamically melting a Mott insulator. Phys. Rev. Lett. 89, 040402 (2002).

27. Volz, T., Dürr, S., Ernst, S., Marte, A. \& Rempe, G. Characterization of elastic scattering near a Feshbach resonance in ${ }^{87} \mathrm{Rb}$. Phys. Rev. A 68, 010702(R) (2003).

\section{Acknowledgements}

We thank W. Ketterle, C. Chin and S. Kokkelmans for valuable discussions. This work was supported by the Austrian Science Fund (FWF) within SFB 15 (project part 17). P.v.d.S. acknowledges support within the ESF-program QUDEDIS during his stay in Innsbruck. P.S.J. was partially supported by the within the ESF-program QU

Correspondence and requests for materials should be addressed to J.H.D

Supplementary Information accompanies this paper on www.nature.com/naturephysics. 